\title{
Dosimetric Effects Due to Inter-Observer Variability of Organ Contouring When Utilizing a Knowledge-Based Planning System for Prostate Cancer
}

\author{
Han Liu*, Christopher Amaloo, Benjamin Sintay, David Wiant \\ Department of Radiation Oncology, Cone Health Cancer Center, Greensboro, USA \\ Email: ^han.liu@conehealth.com
}

How to cite this paper: Liu, H., Amaloo, C., Sintay, B. and Wiant, D. (2021) Dosimetric Effects Due to Inter-Observer Variability of Organ Contouring When Utilizing a Knowledge-Based Planning System for Prostate Cancer. International Journal of Medical Physics, Clinical Engineering and Radiation Oncology, 10, 47-58. https://doi.org/10.4236/ijmpcero.2021.1020 $\underline{05}$

Received: February 23, 2021

Accepted: May 15, 2021

Published: May 18, 2021

Copyright $\odot 2021$ by author(s) and Scientific Research Publishing Inc. This work is licensed under the Creative Commons Attribution International License (CC BY 4.0).

http://creativecommons.org/licenses/by/4.0/ (c) (i) Open Access

\begin{abstract}
Purpose: Radiotherapy is a widely accepted standard of care for early-stage prostate cancer, and it is believed that the plan quality and treatment outcome are associated with contour accuracy of both the target and organs-at-risk (OAR). The purposes of this study are to 1) assess geometric and dosimetric uncertainties due to inter-observer contour variabilities and 2) evaluate the effectiveness of geometric indicators to predict target dosimetry in prostate radiotherapy. Methods: Twenty prostate patients were selected for this retrospective study. Five experienced clinicians created unique structure sets containing prostate, seminal vesicles, bladder, and rectum for each patient. A fully automated script and knowledge-based planning routine were utilized to create standardized and unbiased plans that could be used to evaluate changes in isodose distributions due to inter-observer variability in structure segmentation. Plans were created on a "gold-standard" structure set, as well as on each of the user-defined structure sets. Results: Inter-observer variability of contours during structure segmentation was very low for clearly defined organs such as the bladder but increased for organs without well-defined borders (prostate, seminal vesicles, and rectum). For plans generated with the user-defined structure sets, strong/moderate correlations were observed between the geometric indicators for target structure agreement and target coverage for both low-risk and intermediate-risk patient groups, while OAR indicators showed no correlation to final dosimetry. Conclusions: Target delineation is crucial in order to maintain adequate dosimetric coverage regardless of the associated inter-observer uncertainties in OAR contours that had a limited impact upon final dosimetry.
\end{abstract}




\section{Keywords}

Inter-Observer Variability of Organ Contouring, Knowledge-Based

Treatment Planning, Prostate Radiotherapy

\section{Introduction}

Prostate cancer represents the single most common individual cancer site for men with an estimated 174,650 new cases and 31,620 deaths yearly, which represents over $20 \%$ of the cancer burden for males [1]. Treatment for prostate cancer typically consists of active monitoring, surgery, radiotherapy, or some combination of these approaches [2]. Volumetric modulated arc therapy (VMAT), a new delivery modality, has achieved quick and widespread clinical acceptance as standard of practice for the radiation treatment of prostate cancer due to its improved organ-at-risk (OAR) sparing and high dose conformality to the target, which creates the opportunity for increased daily prescription dose and the improved therapeutic ratio [3] [4]. Studies have shown a correlation between plan quality and patient outcomes, with a definitive benefit to those techniques that support dose escalation while simultaneously limiting high rectal dose to avoid toxicities [5] [6] [7] [8].

Accurate delineation of targets and OAR structures is widely believed to be a foundation of successful radiation treatment on the basis that quality organ segmentation affects patient outcomes [9]. Studies have used different geometric metrics to evaluate contour variability, and have used these as indicators of plan quality [10] [11]. Popular geometric metrics for contour uncertainty evaluation include overlap index (OI), dice similarity coefficient (DSC), and centroid-tocentroid distance $\left(D_{\mathrm{C}}\right)$, etc. [12] [13]. While these metrics are simple to compute, they lack any true clinical meaning, as they do not account for target and OAR interaction nor is there an established correlation with dosimetric effects or patient outcome [14]. One method to better understand the effect contour variation could have on dosimetry and outcome is the dosimetric comparison of calculated radiotherapy treatment plans based upon a user-defined test structure set (TSS) and an expert gold-standard structure set (ESS) [14] [15].

Until recently, the unique nature of creating individual inverse radiation treatment plans made the production of plans onerous and inconsistent, as the plan optimizer could not be systematically and dynamically adjusted without creating a unique, unreproducible result for a given structure set and optimization. Knowledge-based planning (KBP) is a relatively novel development designed to leverage dosimetric and geometric information from previous clinical plans to produce a model which is capable of estimating dose-volume-histogram (DVH) projections for a given set of contours [16] [17] [18]. This technique can reduce plan variation and optimization time in a manner typically associated with advanced treatment planner expertise while reducing planning time and 
producing consistent, high quality, and clinically acceptable treatment plans [19] [20]. Recently, new features of the Eclipse Scripting Application Programming Interface (ESAPI) have made it possible to generate a complete treatment plan without any user interaction [21]. Normally, the decision-making process in treatment planning is highly subjective and remains dependent on the knowledge, experience, and capability of the planner [22] [23]. A previous study showed the combination of KBP and automated scripting has the capability to produce exceptional treatment plans with minimal planner interaction for prostate cancer treatment [21]. The purposes of this study are to 1) investigate the geometric uncertainties of the target and OAR contours due to the inter-observer variability for prostate treatment; 2) obtain dosimetric endpoints from treatment plans automatically generated by a novel KBP based framework for plans that use gold standard structure set (ESS) and test structure sets (TSS) to evaluate the dosimetric impact of inter-observer contour variability on target coverage; 3) evaluate the correlation of contour uncertainty and planning target volume (PTV) dose coverage using the $R^{2}$ statistics.

\section{Methods and Materials}

\subsection{Patient Data}

Twenty prostate cancer patients were selected for this retrospective study. All patients underwent computed tomography (CT) simulation in the supine position with Vac-Lok immobilization for the lower legs and a scan thickness of 2 $\mathrm{mm}$ from mid-abdomen to mid-thigh. The CT scans were imported into the Eclipse treatment planning system (TPS) for planning. A group of 5 experienced clinicians (2 medical dosimetrists, 3 medical physicists) was tasked with the segmentation of the following structures on the planning CT image: the prostate gland, seminal vesicles (SV), bladder, and rectum, utilizing best practices with full access to guidelines and supplemental resources. Each CT structure set was uniquely identified as TSS- 1 to TSS- 5 for each of the 20 patients. ESS was created by a contour imaging specialist who is responsible for all normal tissue segmentation in our clinic and an experienced radiation oncologist who specializes in prostate cancer. The imaging specialist generated the bladder and rectum, while the radiation oncologist delineated the prostate gland and SV. The final structure sets were then reviewed by the radiation oncologist and a clinical physicist for accuracy.

\subsection{Treatment Planning}

Both low-risk patients (LRP) and intermediate-risk patients (IRP) were included in this study, the clinical target volume (CTV) was prostate for LRP, and prostate + SV for IRP. Margins for the PTV were non-isotropic, with a $6 \mathrm{~mm}$ posterior expansion of the CTV and an $8 \mathrm{~mm}$ expansion in all other directions. To investigate the effect of dose distribution changes due to inter-observer contour differences, the following combination of structure sets were used to generate 
treatment plans: 1) $\left.\left.\left.\mathrm{ESS}_{\text {target }}-\mathrm{ESS}_{\mathrm{OAR}} ; 2\right) \mathrm{ESS}_{\text {target }}-\mathrm{TSS}_{\mathrm{OAR}} ; 3\right) \mathrm{TSS}_{\text {target }}-\mathrm{ESS}_{\mathrm{OAR}} ; 4\right) \mathrm{TSS}_{\text {tar- }}$ ${ }_{\text {get }}-\mathrm{TSS}_{\mathrm{OAR}}$. A previously discussed ESAPI based automated planning (AP) routine was used to automatically create treatment plans based on a given combination of structure sets [21]. The workflow of the AP routine is as follows: after a valid CT image and associated structure set are imported into the Eclipse TPS, a script is initiated to create a course, plan, and two VMAT fields (isocenter automatically assigned to the geometric center of the PTV) utilizing 358 degree arcs with collimator rotations of 355 and 85 degrees, respectively. The script generates or utilizes all appropriate names, reference points, prescription information, beam calculation model, and dose calculation setting. The AP routine utilizes a sitespecific KBP model to generate initial DVH estimates for plan optimization. The KBP model was trained with a set of 80 prostate patients. Outliers were determined through separate data analysis and removed to ensure a robust model. Once optimized, the plan undergoes final dose calculation and is normalized such that $100 \%$ of the prescribed dose ( $78 \mathrm{~Gy}$ ) is delivered to $98 \%$ of the PTV volume. The generated plan quality is then automatically evaluated by the AP script against our departmental dosimetric guidelines to ensure sufficient OAR sparing. Additional targeted optimization is conditionally performed and applied by the AP script if any constraint fails, and a final plan is produced once all criteria are met. In this manner, the AP routine was able to generate unbiased treatment plans for the different structure sets without any additional user input, interactive intervention, or post calculation adjustments that would occur with manual planning.

\subsection{Geometric and Dosimetric Evaluation}

The following geometric indices between the test and the gold-standard contour sets are utilized for concordance measures: 1) Centroid-to-centroid distance $\left.\left(D_{C}\right) ; 2\right)$ Overlap index $\left.(O I) ; 3\right)$ Dice similarity coefficient (DSC). The dosimetric indices used for the plan evaluation were $V_{78 G y}$ (percentage of the volume receiving prescription dose) for the PTV, and mean dose $\left(D_{\text {mean }}\right), V_{50 \mathrm{G} y}$, and $V_{60 \mathrm{~Gy}}$ (percentage of volume receiving $50 \mathrm{~Gy}$ and $60 \mathrm{~Gy}$ ) for the OAR. In order to have a fair comparison, all generated plans received identical normalization. Although treatment plans were created with different sets of contours, the dosimetric evaluation was performed on the gold-standard contour sets.

The correlation between the geometric and dosimetric indices for the PTV was evaluated using $R^{2}$ statistics for plans generated with different combinations of structure sets $\left(\mathrm{TSS}_{\text {target }}-\mathrm{ESS}_{\mathrm{OAR}}\right.$ and $\left.\mathrm{TSS}_{\text {target }}-\mathrm{TSS}_{\mathrm{OAR}}\right)$. In general, a correlation coefficient $r$ between -0.2 and 0.2 indicates almost no relationship; $r$ between 0.2 and 0.4 ( -0.4 to -0.2$)$ indicates a weak positive (negative) linear relationship; $r$ between 0.4 and 0.7 ( -0.7 to -0.4$)$ indicates a moderate positive (negative) linear relationship; $r$ between 0.7 and 1 ( -1 to -0.7$)$ indicates a strong positive (negative) linear relationship.

To investigate the impact of target coverage upon the inter-observer contour uncertainty, a paired Student's $t$-test was used to test the PTV dose coverage 
between plans created with $\mathrm{ESS}_{\text {target }}-\mathrm{ESS}_{\mathrm{OAR}}$ and $\mathrm{TSS}_{\text {target }}-\mathrm{ESS}_{\mathrm{OAR}} / \mathrm{TSS}_{\text {target }}-\mathrm{TSS}_{\mathrm{OAR}}$, with statistically significant results based upon a $p$-value $<0.05$. A paired Student's $t$-test was also used to test the difference in OAR dosimetry between plans created with $\mathrm{ESS}_{\mathrm{Target}}-\mathrm{ESS}_{\mathrm{OAR}}$ and $\mathrm{ESS}_{\mathrm{Target}}-\mathrm{TSS}_{\mathrm{OAR}}$.

\section{Results}

\subsection{Patient Statistics}

The average volume for the CTV was $56.2 \pm 18.8 \mathrm{cc}$ (ranged from $28.4 \mathrm{cc}$ to $88.4 \mathrm{cc}$ ) for LRP and $71.4 \pm 23.5 \mathrm{cc}$ (ranged from $34.2 \mathrm{cc}$ to $110.5 \mathrm{cc}$ ) for IRP. Non-uniform margins yielded average PTV volumes of $138.1 \pm 34.3 \mathrm{cc}$ (range from 80.9 to $191.3 \mathrm{cc}$ ) for LRP and $183.6 \pm 43.8 \mathrm{cc}$ (range from 109.4 to 262.7 cc) for IRP. Rectal mean volume was $86.6 \pm 35.9 \mathrm{cc}$ with a range of 45.8 to $172.5 \mathrm{cc}$, while the mean bladder volume was $175.4 \pm 77.1 \mathrm{cc}$, ranging from 83.0 to $386.1 \mathrm{cc}$.

\subsection{Geometric Evaluation}

The geometric evaluation was performed by calculating the $O I, D S C$ and Dc between the test structure sets (TSS-1 to TSS-5) and gold-standard structure sets (ESS). Table 1 lists the average and standard deviation of the various geometric measures of the PTV and OAR for both the LRP and IRP groups. Figure 1 plots

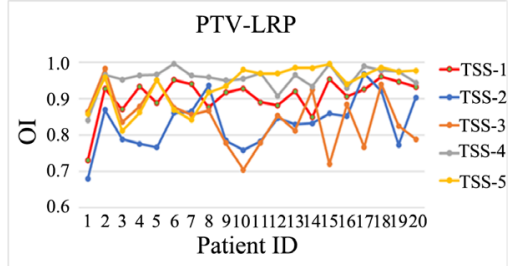

(a)

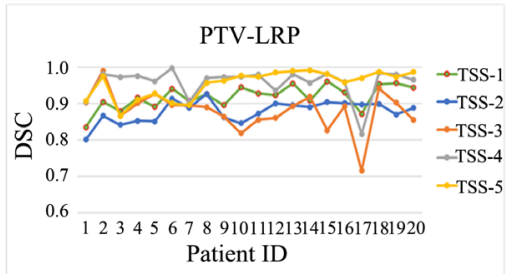

(b)

PTV-LRP

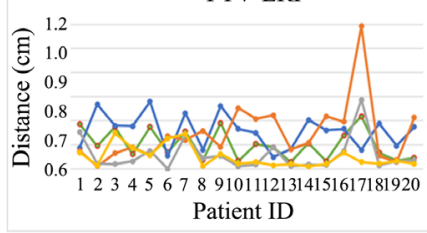

(c)

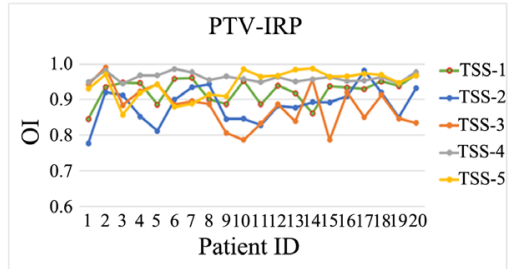

(d)

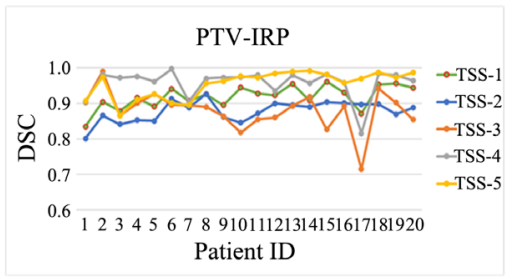

(e)

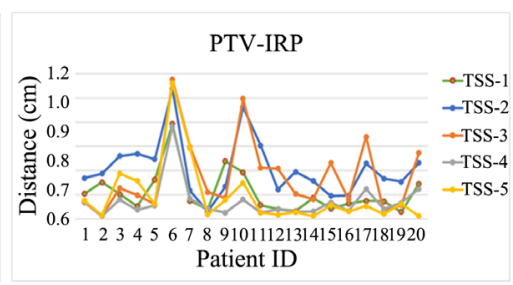

(f)

Figure 1. The overlap index $(O I)$, dice similarity coefficient $(D S C)$ and centroid-tocentroid distance $\left(D_{\mathrm{C}}\right)$ between the gold-standard and test PTVs for low-risk and intermediate-risk patient groups. (a) $O I$ for LRP; (b) DSC for LRP; (c) $D_{C}$ for LRP; (d) $O I$ for IRP; (e) DSC for IRP; (f) $D_{C}$ for IRP. 
Table 1 . The average overlap index (OI), dice similarity coefficient (DSC) and centroid-tocentroid distance $\left(D_{\mathrm{C}}\right)$ for the PTV (LRP and IRP) and organs-at-risk between the goldstandard contour sets (EES) and test contour sets (TSS-1 to TSS-5).

\begin{tabular}{ccccc}
\hline & $\mathrm{PTV}_{\mathrm{LRP}}$ & $\mathrm{PTV}_{\mathrm{IRP}}$ & Bladder & Rectum \\
\hline$O I$ & $0.90 \pm 0.08$ & $0.92 \pm 0.05$ & $0.96 \pm 0.03$ & $0.86 \pm 0.09$ \\
$D S C$ & $0.92 \pm 0.05$ & $0.92 \pm 0.05$ & $0.96 \pm 0.02$ & $0.89 \pm 0.06$ \\
$D_{C}(\mathrm{~cm})$ & $0.21 \pm 0.18$ & $0.26 \pm 0.25$ & $0.09 \pm 0.07$ & $0.41 \pm 0.38$ \\
\hline
\end{tabular}

the $O I, D S C$ and $D_{\mathrm{C}}$ of the PTV for each individual patient for both LRP and IRP groups, and the results for the bladder and rectum are shown in Figure 2. Noticeable geometric variations were found for the PTV (both LRP and IRP) and the rectum contours, while no substantial changes in bladder contours $(<5 \%$ on average) were observed from our data sample.

\subsection{Dosimetric Evaluation}

Figure 3 shows ratios of the PTV dose coverage between test plans $\left(\mathrm{TSS}_{\text {target }} \mathrm{ESS}_{\mathrm{OAR}}\right.$ and $\mathrm{TSS}_{\text {target }}-\mathrm{TSS}_{\mathrm{OAR}}$ ) and the gold-standard plan $\left(\mathrm{ESS}_{\text {target }}-\mathrm{ESS}_{\mathrm{OAR}}\right)$ as a function of various geometric indices for LRP. The $R^{2}$ statistic was used to study the correlation of dosimetric and geometric indices for the PTV dose coverage, and the resulting correlation coefficients $(r)$ are shown in Figure 3 as well. Strong correlations between the PTV coverage and OI/DSC were observed for all test plans studied $(r>0.7)$, while moderate correlations were found between the PTV coverage and $D_{\mathrm{C}}$. The dosimetric and geometric correlations were also investigated for IRP, as shown in Figure 4. Strong correlations between the PTV coverage and $O I$ were observed $(r>0.7)$, while moderate correlations were found between the PTV coverage and $D S C / D_{C}$

In this study we performed a paired Student's $t$-test to investigate the statistical significance of the PTV coverage between plans created with ESS $_{\text {target }}-\mathrm{ESS}_{\mathrm{OAR}}$ and $\mathrm{TSS}_{\text {target }}-\mathrm{ESS}_{\mathrm{OAR}} / \mathrm{TSS}_{\text {target }}-\mathrm{TSS}_{\mathrm{OAR}}$. Compared to plans created with $\mathrm{ESS}_{\text {target }^{-}}$ ESS $_{\text {OAR }}$, statistically significant PTV dose reductions were found for plans created with TSS $_{\text {target }}-$ ESS $_{\text {OAR }}$ and TSS target $^{-}$TSS $_{\text {OAR }}$ for both LRP and IRP groups $(p \ll$ 0.001 ), indicating that the inter-observer uncertainties in target delineation play an important role in achieving a high-quality treatment plan, and as such may affect the treatment outcome.

A paired Student's $t$-test was also used to test the OAR dose differences between plans created with $\mathrm{ESS}_{\text {Target }}-\mathrm{ESS}_{\mathrm{OAR}}$ and $\mathrm{ESS}_{\text {Target }} \mathrm{TSS}_{\mathrm{OAR}}$, the resulting $p$ values are listed in Table 2 for LRP, and Table 3 for IRP, respectively. No statistically significant differences were observed in terms of OAR dosimetry (using $D_{\text {mean }}, V_{50 \mathrm{~Gy}}$ and $V_{60 \mathrm{~Gy}}$ as indicators) for both LRP and IRP groups ( $\left.p>0.05\right)$, which indicates the precision delineation of the bladder and rectum contours may not be necessary to achieve treatment plan with similar qualities.

\section{Discussions}

It is believed that the accurate contour delineation of the target and organs-at-risk 


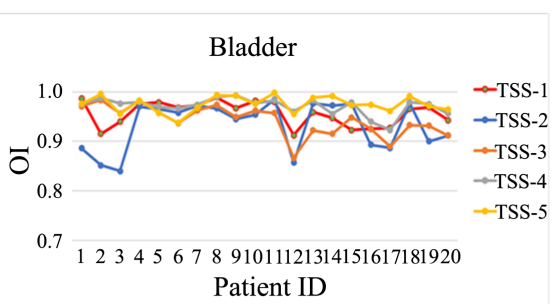

(a)

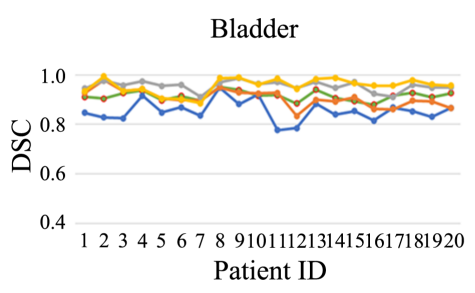

(b)

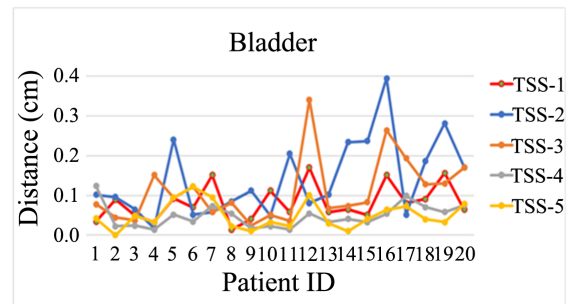

(c)

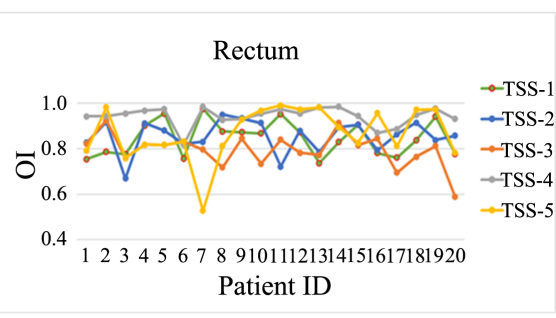

(d)

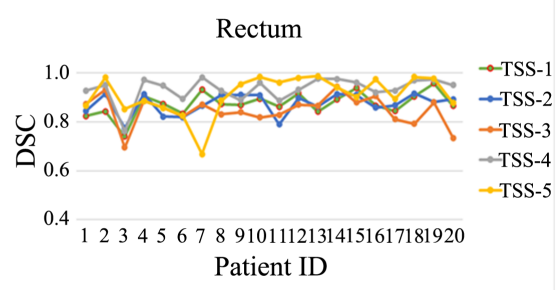

(e)

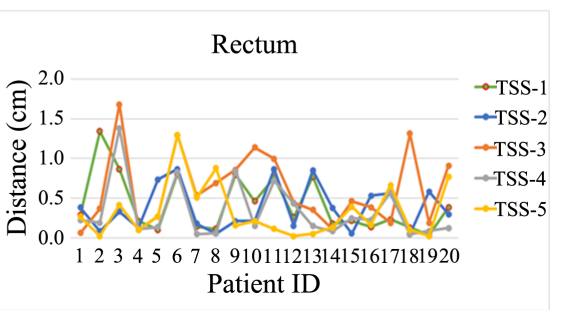

(f)

Figure 2. The overlap index $(O I)$, dice similarity coefficient $(D S C)$ and centroid-tocentroid distance $\left(D_{\mathrm{C}}\right)$ between the gold-standard and test OARs. (a) OI for bladder; (b) $D S C$ for bladder; (c) $D_{C}$ for bladder; (d) $O I$ for rectum; (e) $D S C$ for rectum; (f) $D_{C}$ for rectum.

Table 2. $p$-values from paried student's $t$-test for organs-at-risk (OAR) between plans created with $\mathrm{EES}_{\text {Target }}-\mathrm{ESS}_{\mathrm{OAR}}$ and $\mathrm{EES}_{\text {Target }}-\mathrm{TSS}_{\mathrm{OAR}}$ structure sets for low-risk patient (LRP) group.

\begin{tabular}{ccccccc}
\hline \multirow{3}{*}{ Bladder } & & TSS-1 & TSS-2 & TSS-3 & TSS-4 & TSS-5 \\
\hline \multirow{3}{*}{ Rectum } & $D_{\text {mean }}$ & 0.17 & 0.83 & 0.12 & 0.96 & 0.75 \\
& $V_{50 \mathrm{~Gy}}$ & 0.08 & 0.78 & 0.14 & 0.59 & 0.66 \\
& $V_{60 \mathrm{~Gy}}$ & 0.08 & 0.82 & 0.16 & 0.55 & 0.94 \\
\hline & $D_{\text {mean }}$ & 0.79 & 0.10 & 0.45 & 0.19 & 0.04 \\
& $V_{50 \mathrm{~Gy}}$ & 0.71 & 0.64 & 0.61 & 0.27 & 0.08 \\
& $V_{60 \mathrm{~Gy}}$ & 0.49 & 0.42 & 0.76 & 0.19 & 0.06 \\
\hline
\end{tabular}

Table 3. $p$-values from paried student's $t$-test for organs-at-risk (OAR) between plans created with $\mathrm{EES}_{\mathrm{Target}}-\mathrm{ESS}_{\mathrm{OAR}}$ and $\mathrm{EES}_{\text {Target }}-\mathrm{TSS}_{\mathrm{OAR}}$ structure sets for intermediate-risk patient (IRP) group.

\begin{tabular}{ccccccc}
\hline \multirow{3}{*}{ Bladder } & & TSS-1 & TSS-2 & TSS-3 & TSS-4 & TSS-5 \\
& $D_{\text {mean }}$ & 0.33 & 0.28 & 0.77 & 0.08 & 0.92 \\
& $V_{50 \text { Gy }}$ & 0.10 & 0.95 & 0.06 & 0.46 & 0.27 \\
& $V_{60 G y}$ & 0.11 & 0.57 & 0.06 & 0.31 & 0.22 \\
\hline \multirow{3}{*}{ Rectum } & $D_{\text {mean }}$ & 0.99 & 0.49 & 0.95 & 0.15 & 0.88 \\
& $V_{50 \text { Gy }}$ & 0.52 & 0.96 & 0.61 & 0.13 & 0.88 \\
& $V_{60 \mathrm{~Gy}}$ & 0.35 & 1.00 & 0.54 & 0.17 & 0.81 \\
\hline
\end{tabular}




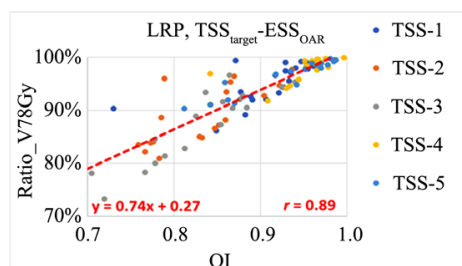

(a)

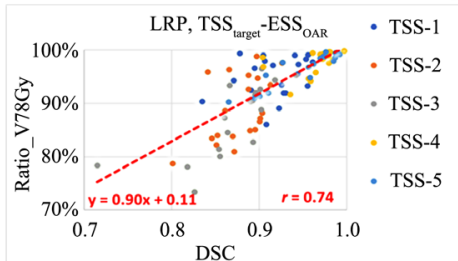

(b)

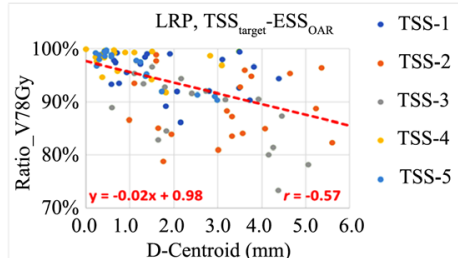

(c)

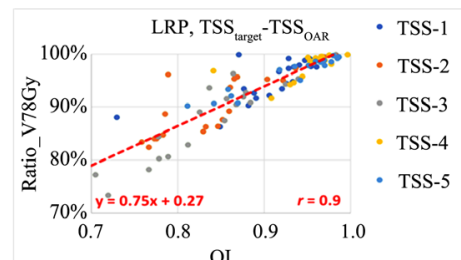

(d)

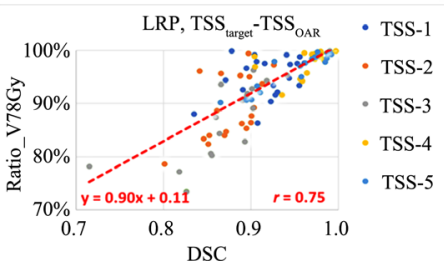

(e)

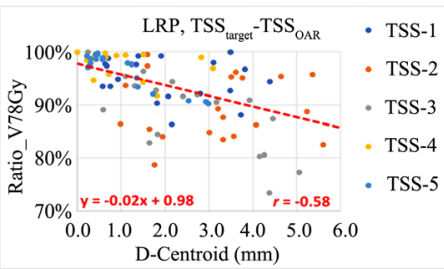

(f)

Figure 3. Correlation of the PTV coverages and geometric indices for low-risk patient (LRP) group. The PTV coverages were expressed as a ratio of test plan dose to goldstandard dose. The linear regression analysis is performed and shows the dashed lines together with fitted equations.

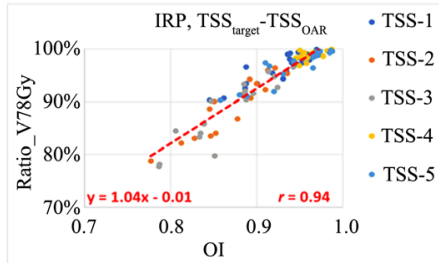

(a)

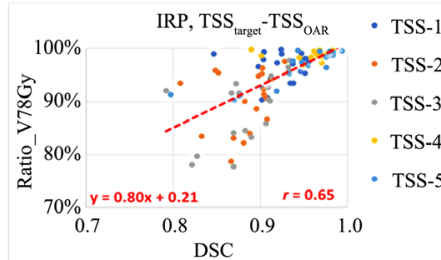

(b)

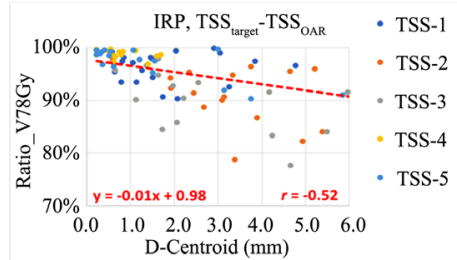

(c)

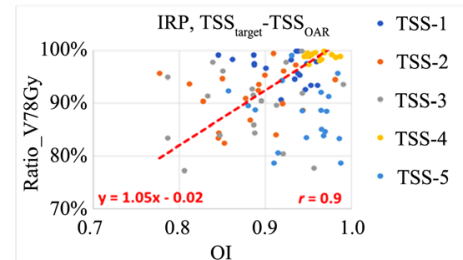

(d)

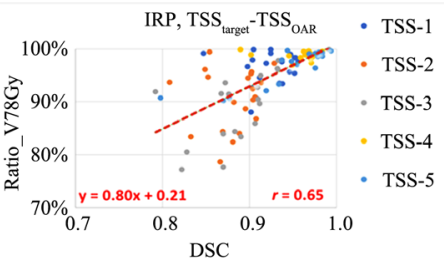

(e)

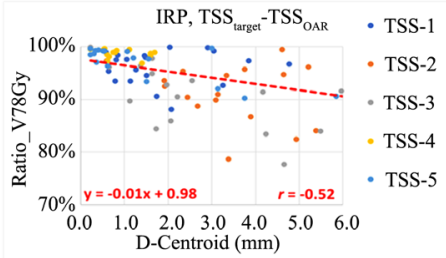

(f)

Figure 4. Correlation of the PTV coverages and geometric indices for intermediate-risk patient (IRP) group. The PTV coverages were expressed as a ratio of test plan dose to gold-standard dose. The linear regression analysis is performed and shows dashed lines together with fitted equations. 
is essential in creating high-quality treatment plans for radiotherapy. However, manual contouring by clinicians, hereby referred to as "precision contouring", is a time- and labor-intensive process, and subject to inter- and intra-observer variability. In this study we intended to focus on the inter-observer geometric uncertainties in structure definition of the target and OAR contours in prostate radiotherapy, and to investigate the impact of these inter-observer contour variances on target and OAR dosimetry. We found bladder segmentation to be very consistent between all clinicians (within 5\%) due to it having well-defined borders which show clearly on planning CT scans and aid in structure delineation. Both geometric indicators $(O I$ and $D S C$ ) showed greater variabilities for the target and rectal contours $(\sim 10 \%)$. This can be attributed to poor contrast between the region of interest and surrounding tissues.

The utilization of an automated planning routine is vital to this evaluation as it allowed us to generate unbiased high-quality treatment plans based upon a given structure set. The average time to produce a single plan from start to finish was approximately 20 minutes. Additionally, the AP script is capable of creating plans for 10 patients simultaneously. The AP routine does not require additional input from the planner on either the front or back end, which reduces uncertainties associated with the individual planner's knowledge and experience.

The goal when developing a treatment plan in radiotherapy is to achieve adequate target coverage while sparing normal tissue as much as possible. PTV coverage was dosimetrically equivalent between plans created with the same goldstandard target contours ( $\mathrm{ESS}_{\text {target }}$ ) but different OAR contours ( $\mathrm{ESS}_{\mathrm{OAR}}$ and $\mathrm{TSS}_{\mathrm{OAR}}$ ). This was intuitively coherent as the AP planning routine accessed the same KBP model and used the same target contour, and the same plan normalization was used (100\% of prescribed dose delivered to $98 \%$ of the PTV volume). However, compared with plans created with $\mathrm{ESS}_{\text {target }}-\mathrm{ESS}_{\mathrm{OAR}}$, statistically significant reductions of the PTV coverage were found for plans created with $\mathrm{TSS}_{\text {target }^{-}}$ $\mathrm{ESS}_{\mathrm{OAR}}$ and $\mathrm{TSS}_{\text {target }}-\mathrm{TSS}_{\mathrm{OAR}}(p \ll 0.001$ from paired Student's $t$-test). This was because the plan was normalized to $\mathrm{TSS}_{\text {Target }}$ during the planning process, while all the dosimetric analysis was performed for ESS $_{\text {target }}$. The target under-coverage was purely due to the geometric variations between the $\mathrm{ESS}_{\text {target }}$ and $\mathrm{TSS}_{\text {target. }}$. For both the LRP and IRP groups, strong/moderate correlations were observed between the PTV coverage and geometric indices ( $O I, D S C$ and $D_{\mathrm{C}}$ ), which can be explained by the fact that the closer the test PTVs are to the gold-standard PTVs, the better the target coverage. In this study we did not compare OAR dosimetry for plans created with $\mathrm{TSS}_{\text {target }}$ and $\mathrm{ESS}_{\text {target}}$, because OAR dose comparisons are of little use if the target coverages are not adequate. The OAR dosimetry comparisons between plans using the same target but different OAR structures were performed, and no statistically significant differences were observed for both bladder and rectum for both the LRP and IRP groups (Table 2 and Table 3, $p>$ 0.05 from paired Student's $t$-test). This is an indication that if a certain level of accuracy in bladder and rectum delineation is reached ( $~ 10 \%$ in our study), our automated planning routine can achieve similar plan quality for prostate treat- 
ment. Tight conformation of high dose to the target associated with VMAT planning helps the understanding of how changes in target definition will have a major impact on the plan quality, while the OAR dose appears to be relatively independent of moderate contour variations.

Attention must be given to the transfer of this work to other more complex disease sites. Compared to sites such as head and neck, prostate treatment planning considers relatively few critical OAR such as the bladder and rectum, both parallel organs with an emphasis on reducing the mid to higher dose levels. Furthermore, the positional relationship of the target to this OAR is relatively consistent across the prostate patients, which could be the reason why precision contouring of OAR has a minimum impact on the plan quality. More complicated anatomy, critical structures, geometric relationship between target and OAR, and serial organ involvement may produce different dosimetric and geometric interactions compared with those examined in this study.

\section{Conclusions}

Inter-observer variability of contours during structure segmentation was very low (less than $5 \%$ on average) for clearly defined organs such as the bladder but increased for organs with less well-defined borders (prostate, seminal vesicles, and rectum). The fully automated AP script routine was successfully utilized in the creation of standardized and unbiased plans for the evaluation of isodose distributions based upon contour disparity due to inter-observer variability. All the plans generated were normalized in such a way that $100 \%$ of the prescribed doses were delivered to $98 \%$ of the PTV volume, thus target coverage of plans created with $\mathrm{ESS}_{\text {target }}-\mathrm{TSS}_{\mathrm{OAR}}$ was guaranteed. However, that is not the case for plans created with TSS $_{\text {target }}$. Strong/moderate correlations were observed between the PTV coverage and geometric indices ( $O I, D S C$, and $\left.D_{\mathrm{C}}\right)$ for both the LRP and IRP groups, which is an indication that the closer the test PTVs are to the gold-standard PTVs, the better the target coverage is. For both the low-risk and intermediate-risk patient groups, the level of target delineation accuracy is crucial in order to maintain adequate dosimetric coverage, while there is the limited impact of inter-observer OAR segmentation in reference to final OAR dosimetry.

Thus, we propose that there may be limited benefit from human-based OAR contouring in prostate radiotherapy. It is recognized that this result is specific to the prostate and requires further study for more complex treatment sites.

\section{Conflicts of Interest}

This research was supported by a grant from Varian Medical Systems, Palo Alto, CA.

\section{References}

[1] Siegel, R.L., Miller, K.D. and Jemal, A. (2019) Cancer Statistics. CA: A Cancer Journal for Clinicians, 69, 7-34. https://doi.org/10.3322/caac.21551 
[2] Hamdy, F.C., Donovan, J.L., Lane, J., Mason, M., Metcalfe, C., Holding, P., Davis, M., Peters, T.J., Turner, E.L. and Martin, R.M. (2016) 10-Year Outcomes after Monitoring, Surgery, or Radiotherapy for Localized Prostate Cancer. The New England Journal of Medicine, 375, 1415-1424. https://doi.org/10.1056/NEJMoa1606220

[3] Yoo, S., Wu, Q.J., Lee, W.R. and Yin, F.-F. (2010) Radiotherapy Treatment Plans with RapidArc for Prostate Cancer Involving Seminal Vesicles and Lymph Nodes. International Journal of Radiation Oncology Biology Physics, 76, 935-942. https://doi.org/10.1016/j.ijrobp.2009.07.1677

[4] Palma, D., Vollans, E., James, K., Nakano, S., Moiseenko, V., Shaffer, R., McKenzie, M., Morris, J. and Otto, K. (2008) Volumetric Modulated arc Therapy for Delivery of Prostate Radiotherapy: Comparison with Intensity-Modulated Radiotherapy and Three-Dimensional Conformal Radiotherapy. International Journal of Radiation Oncology Biology Physics, 72, 996-1001.

https://doi.org/10.1016/j.ijrobp.2008.02.047

[5] Zelefsky, M.J., Fuks, Z., Hunt, M., Yamada, Y., Marion, C., Ling, C.C., Amols, H., Venkatraman, E.S. and Leibel, S.A. (2002) High-Dose Intensity Modulated Radiation Therapy for Prostate Cancer: Early Toxicity and Biochemical Outcome in 772 Patients. International Journal of Radiation Oncology Biology Physics, 53, 11111116. https://doi.org/10.1016/S0360-3016(02)02857-2

[6] Pollack, A., Zagars, G.K., Starkschall, G., Antolak, J.A., Lee, J.J., Huang, E., Von Eschenbach, A.C., Kuban, D.A. and Rosen, I. (2002) Prostate Cancer Radiation Dose Response: Results of the M.D. Anderson Phase III Randomized Trial. International Journal of Radiation Oncology Biology Physics, 53, 1097-1105. https://doi.org/10.1016/S0360-3016(02)02829-8

[7] Pollack, A., Hanlon, A.L., Horwitz, E.M., Feigenberg, S.J., Uzzo, R.G. and Hanks, G.E. (2004) Prostate Cancer Radiotherapy Dose Response: An Update of the Fox Chase Experience. The Journal of Urology, 171, 1132-1136.

https://doi.org/10.1097/01.ju.0000111844.95024.74

[8] Moore, K.L., Schmidt, R., Moiseenko, V., Olsen, L.A., Tan, J., Xiao, Y., Galvin, J., Pugh, S., Seider, M.J. and Dicker, A.P. (2015) Quantifying Unnecessary Normal Tissue Complication Risks Due to Suboptimal Planning: A Secondary Study of RTOG 0126. International Journal of Radiation Oncology Biology Physics, 92, 228235. https://doi.org/10.1016/j.ijrobp.2015.01.046

[9] Fiorino, C., Reni, M., Bolognesi, A., Cattaneo, G.M. and Calandrino, R. (1998) Intra- and Inter-Observer Variability in Contouring Prostate and Seminal Vesicles: Implications for Conformal Treatment Planning. Radiotherapy and Oncology, 47, 285-292. https://doi.org/10.1016/S0167-8140(98)00021-8

[10] Lee, W.R., Roach III, M., Michalski, J., Moran, B. and Beyer, D. (2002) Interobserver Variability Leads to Significant Differences in Quantifiers of Prostate Implant Adequacy. International Journal of Radiation Oncology Biology Physics, 54, 457461. https://doi.org/10.1016/S0360-3016(02)02950-4

[11] Vinod, S.K., Min, M., Jameson, M.G. and Holloway, L.C. (2016) A Review of Interventions to Reduce Inter-Observer Variability in Volume Delineation in Radiation Oncology. Journal of Medical Imaging and Radiation Oncology, 60, 393-406. https://doi.org/10.1111/1754-9485.12462

[12] Hanna, G., Hounsell, A. and O’Sullivan, J. (2010) Geometrical Analysis of Radiotherapy Target Volume Delineation: A Systematic Review of Reported Comparison Methods. Clinical Oncology, 22, 515-525. https://doi.org/10.1016/j.clon.2010.05.006

[13] Jameson, M.G., Holloway, L.C., Vial, P.J., Vinod, S.K. and Metcalfe, P.E. (2010) A 
Review of Methods of Analysis in Contouring Studies for Radiation Oncology. Journal of Medical Imaging and Radiation Oncology, 54, 401-410. https://doi.org/10.1111/j.1754-9485.2010.02192.x

[14] Lim, T.Y., Gillespie, E., Murphy, J. and Moore, K.L. (2019) Clinically Oriented Contour Evaluation Using Dosimetric Indices Generated from Automated Knowledge-Based Planning. International Journal of Radiation Oncology Biology Physics, 103, 1251-1260. https://doi.org/10.1016/j.ijrobp.2018.11.048

[15] Tsuji, S.Y., Hwang, A., Weinberg, V., Yom, S.S., Quivey, J.M. and Xia, P. (2010) Dosimetric Evaluation of Automatic Segmentation for Adaptive IMRT for HeadAnd-Neck Cancer. International Journal of Radiation Oncology Biology Physics, 77, 707-714. https://doi.org/10.1016/j.ijrobp.2009.06.012

[16] Nawa, K., Haga, A., Nomoto, A., Sarmiento, R.A., Shiraishi, K., Yamashita, H. and Nakagawa, K. (2017) Evaluation of a Commercial Automatic Treatment Planning System for Prostate Cancers. Medical Dosimetry, 42, 203-209. https://doi.org/10.1016/j.meddos.2017.03.004

[17] Appenzoller, L.M., Michalski, J.M., Thorstad, W.L., Mutic, S. and Moore, K.L. (2012) Predicting Dose-Volume Histograms for Organs-At-Risk in IMRT Planning. Medical Physics, 39, 7446-7461. https://doi.org/10.1118/1.4761864

[18] Olsen, L.A., Robinson, C.G., He, G.R., Wooten, H.O., Yaddanapudi, S., Mutic, S., Yang, D. and Moore, K.L. (2014) Automated Radiation Therapy Treatment Plan Workflow Using a Commercial Application Programming Interface. Practical Radiation Oncology, 4, 358-367. https://doi.org/10.1016/j.prro.2013.11.007

[19] Hussein, M., South, C.P., Barry, M.A., Adams, E.J., Jordan, T.J., Stewart, A.J. and Nisbet, A. (2016) Clinical Validation and Benchmarking of Knowledge-Based IMRT and VMAT Treatment Planning in Pelvic Anatomy. Radiotherapy and Oncology, 120, 473-479. https://doi.org/10.1016/j.radonc.2016.06.022

[20] Kubo, K., Monzen, H., Ishii, K., Tamura, M., Kawamorita, R., Sumida, I., Mizuno, H. and Nishimura, Y. (2017) Dosimetric Comparison of RapidPlan and Manually Optimized Plans in Volumetric Modulated Arc Therapy for Prostate Cancer. Physica Medica, 44, 199-204. https://doi.org/10.1016/j.ejmp.2017.06.026

[21] Amaloo, C., Hayes, L., Manning, M., Liu, H. and Wiant, D. (2019) Can Automated Treatment Plans Gain Traction in the Clinic? Journal of Applied Clinical Medical Physics, 20, 29-35. https://doi.org/10.1002/acm2.12674

[22] Al-Mamgani, A., Heemsbergen, W.D., Peeters, S.T. and Lebesque, J.V. (2009) Role of Intensity-Modulated Radiotherapy in Reducing Toxicity in Dose Escalation for Localized Prostate Cancer. International Journal of Radiation Oncology Biology Physics, 73, 685-691. https://doi.org/10.1016/j.ijrobp.2008.04.063

[23] Caine, H., Whalley, D., Kneebone, A., McCloud, P. and Eade, T. (2016) Using Individual Patient Anatomy to Predict Protocol Compliance for Prostate IntensityModulated Radiotherapy. Medical Dosimetry, 41, 70-74.

https://doi.org/10.1016/j.meddos.2015.08.005 